\title{
THERMOELASTICALLY ACTUATED ACOUSTIC PROXIMITY SENSOR WITH INTEGRATED THROUGH-WAFER INTERCONNECTS
}

\author{
Venkataraman Chandrasekaran ${ }^{1}$, Eugene M. Chow ${ }^{2}$, Thomas W. Kenny* ${ }^{* 1}$, Toshikazu Nishida ${ }^{2}$, \\ Louis N. Cattafesta ${ }^{1}$, Bhavani V. Sankar ${ }^{1}$ and Mark Sheplak ${ }^{1}$ \\ ${ }^{1}$ Department of Aerospace Engineering, Mechanics and Engineering Science \\ ${ }^{2}$ Department of Electrical and Computer Engineering \\ University of Florida \\ Gainesville, FL 32611-6250 \\ ${ }^{* 1}$ Department of Mechanical Engineering \\ *2Department of Electrical and Computer Engineering \\ Stanford University \\ Stanford, CA 94305-4085
}

\begin{abstract}
This paper presents the development of a micromachined acoustic sensor/actuator for real-time cavity monitoring for highspeed, supercavitating vehicles (HSSV). Low-resistance polysilicon through-wafer electrical interconnects have been integrated with the sensor to enable backside contacts for drive and sense circuitry. The sensor and interconnects were fabricated in a CMOS compatible process using a deep reactive ion etch (DRIE), producing a $1 \mathrm{~mm}$ diameter, $10 \mu \mathrm{m}$ thick diaphragm and $20 \mu \mathrm{m}$ diameter high aspect ratio through-wafer vias on a SOI wafer. The diaphragm incorporates a central resistive heater for thermoelastic actuation and diffused piezoresistors for sensing acoustic pressure perturbations. Measured results indicate an acoustic transmitting sensitivity of $22 \mathrm{mPa} / \mathrm{V}$ at a distance of $10 \mathrm{~mm}$ and a frequency of $60 \mathrm{kHz}$, a receiving sensitivity of $0.98 \mu \mathrm{V} /(\mathrm{V} . \mathrm{Pa})$, a flat frequency response over the measured range of $1-20 \mathrm{kHz}$, a linear response from 60-140 $d B$, negligible leakage current for the junctionisolated diffused piezoresistors $(<2 n A$ at $-10 \mathrm{~V})$, low interconnect resistance of $14 \Omega$, and a noise floor of $\approx 1.4 \times 10^{-16} V^{2} / \mathrm{Hz}$ at a bias of $9 \mathrm{~V}$, in the frequency range of operation.
\end{abstract}

\section{INTRODUCTION}

Our goal is to develop a real-time cavity monitoring system for a high-speed, supercavitating underwater vehicle. The high velocity of the vehicle is made possible by supercavitation, which results in the entire vehicle being encapsulated in a gaseous cavity or bubble, thus greatly reducing wetted friction drag [1]. There are several methods to measure the cavity distance, all involving the radiation of energy from the hull towards the cavity interface, the subsequent reflection of the incident wave at the gas/water interface, and the detection of the reflected pulse (Figure 1). The unstable nature and poor electromagnetic (EM) reflection coefficient of the air/water interface are some of the disadvantages of using laser or EM-based techniques. Acoustic techniques possess good signal-to-noise characteristics due to the sound hard boundary at the interface [2]. In addition, as the cavity structure begins to degrade and the interface possesses waviness or bubbly regions, the specular reflection assumption is strongly dependent on the interface roughness relative to the wavelength $(\lambda)$ of the radiated energy. If the wavelength is on the order of the interface roughness or smaller, the incident radiation will be scattered at the wavy interface and may not be redirected to the fixed receiver location. In these regions, acoustic-based techniques provide an advantage because it is possible to fabricate compact devices to radiate acoustic energy possessing wavelengths on the order of a millimeter [3]. Thus acoustic techniques present a potentially efficient way of measuring the interface proximity as well as discerning the nature of the interface. The harsh seawater environment necessitates a sensor possessing a hydrophobic barrier and backside electrical connections (Figure 1).

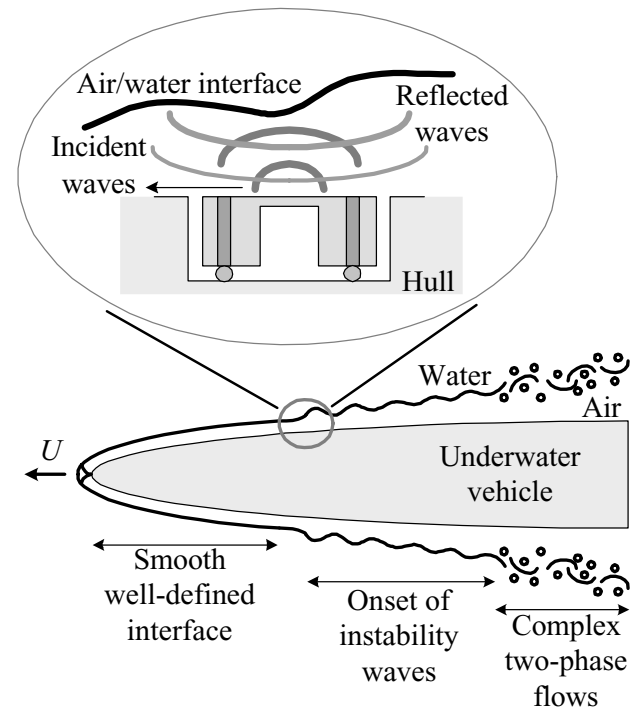

Figure 1: Bump-bonded sensor package placed on the hull of an underwater supercavitating vehicle for monitoring the state and thickness of the air/water interface.

Acoustic proximity sensors based on various operating principles have been reported including thermoelastic [3], capacitive [4] and piezoelectric [5], but these do not meet all the requirements of a real-time cavity monitor in terms of operating frequencies and packaging suitable for harsh environments.

\section{ACOUSTIC PROXIMITY SENSOR}

The acoustic transceiver structure integrates diffused resistors for thermoelastic actuation and piezoresistive detection and borondoped polysilicon electrical through-wafer interconnects (ETWI). Figure 2 shows a top view microscopic image of the sensor/actuator, and a cross-sectional schematic is shown in Figure

Travel support has been generously provided by the Transducers Research Foundation and by the DARPA MEMS and DARPA BioFlips programs. 
3. The device structure consists of a $1 \mathrm{~mm}$ diameter, $10 \mu \mathrm{m}$ thick circular composite diaphragm. Two semicircular diffused heaters (60 $\mu \mathrm{m}$ diameter) are located in the center for electrothermal actuation of the diaphragm. Four diffused p-type silicon piezoresistors are located at the edge of the diaphragm for piezoresistive detection of the membrane vibrations.

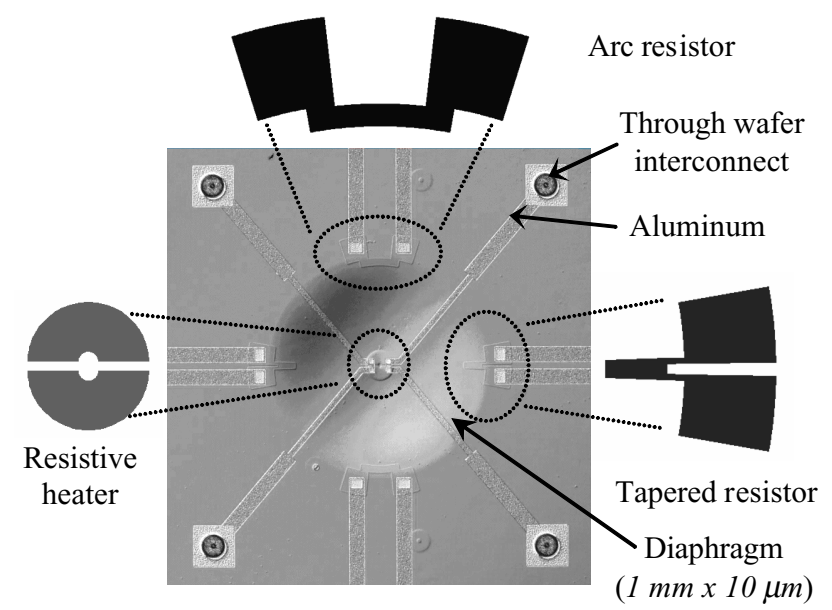

Figure 2: Top view microscopic (diffraction interference contrast) image of the acoustic proximity sensor with integrated throughwafer interconnects.

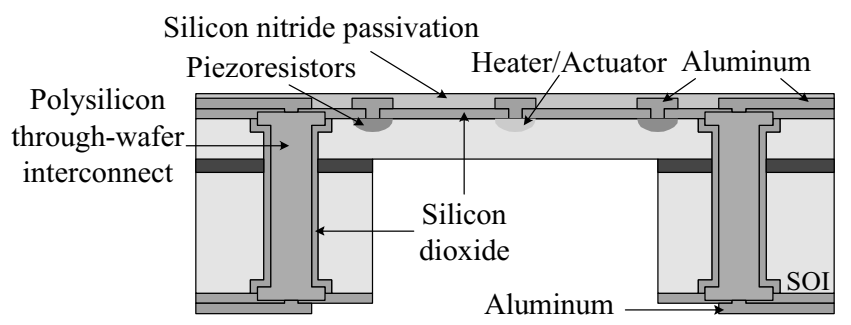

Figure 3: Cross-sectional schematic of the proximity sensor.

The intended application of the sensor necessitates a rugged package capable of withstanding the harsh seawater environment. Therefore, polysilicon ETWI measuring $20 \mu \mathrm{m}$ in diameter have been integrated with the sensor/actuator [6,7]. The use of p-type ETWI enables direct ohmic contact to p-type piezoresistors employed for the acoustic sensor. The ETWI thus enables an integrated "bump-bonded" sensor package with the drive and sense circuitry hidden from the environment. We have previously integrated ETWI with released piezoresistive sensors for cantilever arrays [8]. The heater for thermoelastic actuation and piezoresistors are connected to the ETWI via $75 \mu \mathrm{m}$ wide, $1.4 \mu \mathrm{m}$ thick aluminum traces. A $7000 \AA$ thick silicon dioxide film is thermally grown on top of the silicon layer to provide compressive stress to the diaphragm in addition to serving as a dielectric passivation for the piezoresistors. A $3500 \AA$ low-stress, PECVD silicon nitride passivation layer provides a protective moisture barrier on the top surface of the device.

\section{OPERATING PRINCIPLES}

\section{Thermoelastic Actuation}

Dynamic Joule heating of the resistor causes the formation of a temperature gradient across the diaphragm cross-section. This non-uniform temperature distribution produces a thermal moment that results in out-of-plane bending of the diaphragm. The asymmetrical composite lay-up of the diaphragm (see Figure 3) further enhances the coupling between in-plane forces generated by the heating and the transverse deflection [9]. Thus, by applying a time-varying instantaneous voltage signal, the diaphragm is forced into vibration. The temperature distribution in the diaphragm is a function of the driving frequency of the signal and depends on the depth of penetration of the thermal wave, governed by Fourier's law of heat conduction [10]. The harmonic heating excites the diaphragm at the driving frequency, $\omega$, but also at twice that frequency, $2 \omega$, and at dc. This is due to the non-linear nature of thermoelastic actuation, where the input power has a quadratic dependence on the excitation voltage, causing the power to be redistributed into two frequency bins (i.e., 0 and $2 \omega$ ). By adding a conditioning dc voltage [3] the ratio of power between the two frequencies can be controlled as shown by the following equation,

$$
\left(\bar{V}+V^{\prime} \sin \omega t\right)^{2}=\bar{V}^{2}+\frac{V^{\prime 2}}{2}(1-\cos 2 \omega t)+2 \bar{V} V^{\prime} \sin \omega t
$$

However, adding an offset voltage to the harmonic signal adds a static temperature distribution, which consequently changes the inplane stress field and the resonant frequency of the diaphragm.

\section{Piezoresistive Detection}

Electromechanical transduction of the membrane vibration is achieved via four diffused silicon piezoresistors located at the edge of the diaphragm in a Wheatstone bridge configuration [11]. The arrangement consists of two tapered and two arc-shaped piezoresistors. The piezoresistors are designed such that they possess the same nominal resistance. An acoustic wave impinging on the diaphragm causes it to deflect and changes the stress in the diaphragm. The change in resistance of the piezoresistors due to a change in the stress field is equal but opposite for the arc and tapered resistors due to the nature of the stress being detected. Thus for an undeflected diaphragm, the output of the Wheatstone bridge is zero, but a deflection caused by an acoustic wave produces a differential voltage output across the bridge.

\section{FABRICATION}

The fabrication process begins with the creation of the ETWI [6,7]. After the wafer with ETWI is planarized, the ETWI wafer provides the substrate for the subsequent backend CMOS process to fabricate the acoustic proximity sensor. Figure 4 outlines the overall fabrication sequence.

\section{Electrical Through-Wafer Interconnects}

The fabrication process begins with a $450 \mu \mathrm{m}$ thick double side polished n-type silicon-on-insulator wafer. A $2 \mu m$ thick thermally grown silicon dioxide acts as the mask for the throughwafer via DRIE. The front and the backsides of the wafer were etched for approximately equal durations (4A). Etching through both sides of the wafer was done to maintain a high aspect ratio for the structure. To achieve smooth sidewalls and a straight via profile, a 30 minute timed overetch from the front side of the wafer was performed using an etch recipe with higher passivation. The oxide used as a mask was then stripped using a buffered oxide etch (BOE). After the via formation, the interconnect were dielectrically isolated from the bulk silicon substrate by growing a $2 \mu \mathrm{m}$ thermal oxide (4B). Electrical conduction was achieved through the deposition of $2 \mu \mathrm{m}$ LPCVD polysilicon over the oxide (4B). This was followed by boron diffusion doping of the polysilicon for 2 hours at $1000^{\circ} \mathrm{C}$ and then a drive-in anneal at $1000^{\circ} \mathrm{C}$. 
(A)
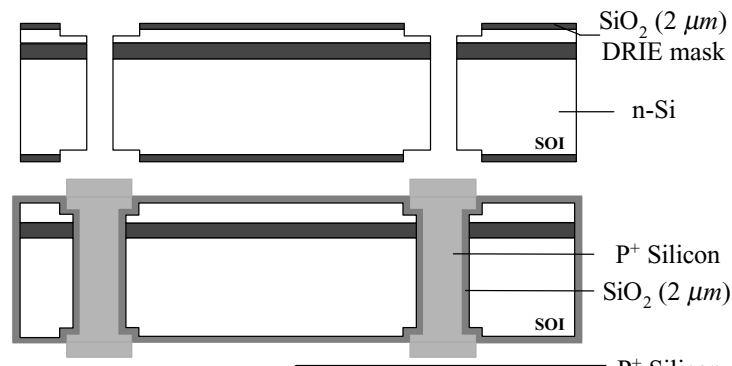

(C)

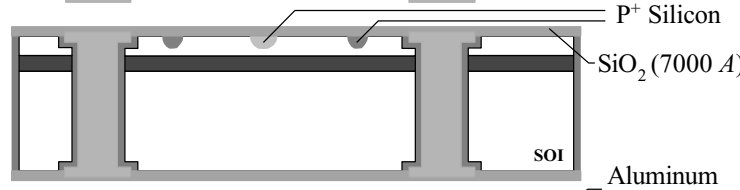

(D)

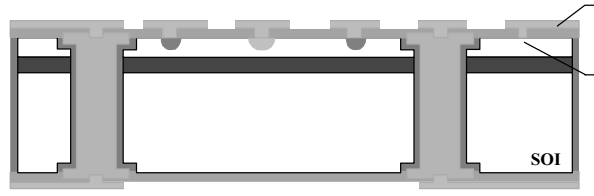

$(1.4 \mu \mathrm{m})$

(E)

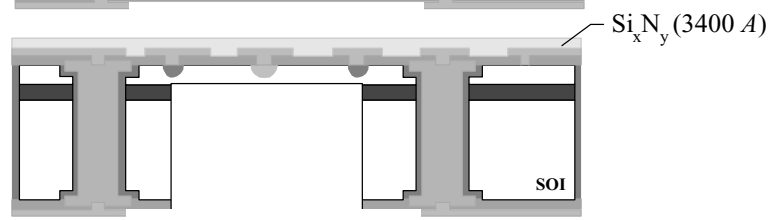

Figure 4: A schematic of the fabrication sequence.

The boron diffusion process results in the formation of a nonconducting borosilicate glass that is not easily etched by hydrofluoric acid. However, a wet oxidation at $1100^{\circ} \mathrm{C}$ for 30 minutes, followed by a 60 minute etch using (6:1) BOE effectively removes the non-conducting glass. The process of polysilicon deposition and boron diffusion doping was repeated 2-3 times to achieve a low-resistance pETWI. Further details of the ETWI fabrication process can be found in Chow et. al [6] and Chandrasekaran et. al [7]. After patterning the ETWI, an isotropic plasma etch was performed on the doped polysilicon to planarize the interconnects and produce a gradually sloping sidewall on the front and back of the wafer (4C). This is critical for the integration of the ETWI with the sensor/actuator since it ensures proper metal coverage over the ETWI.

\section{Acoustic Sensor/Actuator}

A thin layer of oxide was then grown and patterned for the heater implantation. Boron was implanted to achieve $\mathrm{P}^{++}$regions with a concentration of $1 \mathrm{E}-20 \mathrm{~cm}^{-3}$ (4C). After implantation, the wafers were annealed at $1100{ }^{\circ} \mathrm{C}$ for 30 minutes to create a junction depth of $0.75 \mu \mathrm{m}$. The wafers were then patterned with the piezoresistors mask and again implanted with boron to form $\mathrm{P}^{++}$regions of similar concentration (4C). A silicon dioxide layer $7000 A$ thick was thermally grown at $950{ }^{\circ} \mathrm{C}$ to passivate the resistors and provide a compressive stress to the diaphragm (4C). The diffusion of the heater resistors and piezoresistors were simulated using FLOOPS [12] to account for the entire thermal budget of the process flow. A $1.4 \mu m$ thick aluminum $(\mathrm{Al} / 1 \% \mathrm{Si})$ layer was sputtered and patterned once the contact cuts in the oxide dielectric layer were made (4D). Low stress PECVD nitride (3500 $A$ ) was then deposited to form a moisture barrier (4E). The wafers were then patterned on the backside with front to back alignment to create the diaphragm. The relative alignment of the piezoresistors and the diaphragm is critical to ensure piezoresistor placement at the edge of the diaphragm. DRIE was performed from the backside of the wafer up to the buried oxide layer (BOX) (4E).
Once the diaphragm was created, the buried oxide layer was removed using 6:1 BOE.

\section{RESULTS AND DISCUSSION}

Experimental characterization of the sensor/actuator was conducted in the Interdisciplinary Microsystems Laboratory at the University of Florida. The experimental details and results of the electrical and acoustic characterization of the sensor/actuator are described in this section.

\section{Electrical Characterization}

Current versus voltage (I-V) characterizations were performed on the sensors to extract the resistance of the diffused piezoresistors and the ETWI. In addition, I-V measurements were also obtained across the p-n junction formed between the diffused resistors and the silicon substrate to obtain its forward and reverse bias characteristics. The reverse bias characteristics of this $p-n$ junction determines the leakage current from the resistors into the substrate. Minimal leakage is essential for effective Joule heating of the diaphragm and low piezoresistor noise floor. All measurements were made using a Hewlett Packard 4155B semiconductor parameter analyzer and a wafer level probe station.

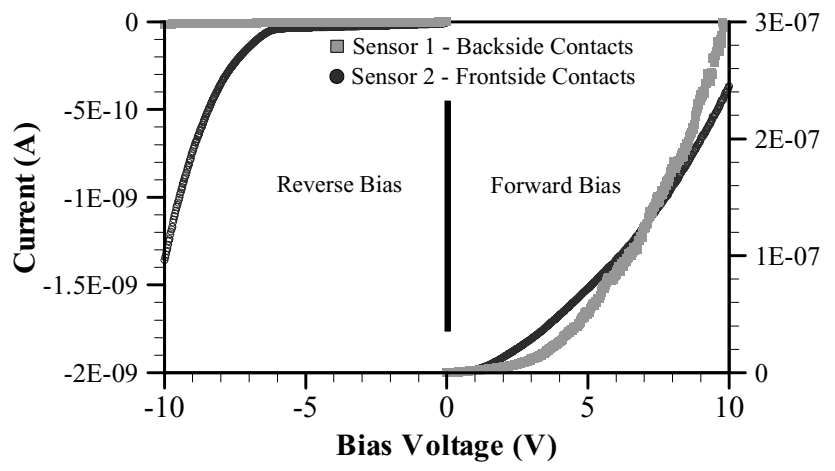

Figure 5: Forward and reverse bias characteristics of the diffused piezoresistors, indicating negligible leakage current $(<2 \mathrm{nA}$ at $10 \mathrm{~V}$ ).

Results of the I-V characterization indicate negligible leakage current up to a reverse bias voltage of $10 \mathrm{~V}$ (Figure 5). The forward and reverse bias characteristics of the $p-n$ junction measured through the ETWI was compared to another sensor with front-side contacts. As indicated in Figure 5, the interconnects do not affect the bias characteristics. The results also indicate deviations from the ideal diode behavior due to a large series resistance of the lightly doped silicon substrate. The average resistance was found to be around $2200 \Omega$ for the actuating heater, $5500 \Omega$ for the arc resistor and $7500 \Omega$ for the tapered resistor. The average ETWI resistance was measured to be around $14 \Omega$.

\section{Diaphragm Surface Velocity Measurements}

Transverse velocity measurements were made on the diaphragm surface using a Polytec scanning laser vibrometer fitted onto an Olympus microscope producing a laser spot size of $2 \mu \mathrm{m}$. The diaphragm was excited using a periodic chirp signal with a specified dc offset. The response of the diaphragm was obtained at specific scan points on the surface. This velocity information measured over the entire surface is then integrated to obtain displacement mode shapes. 

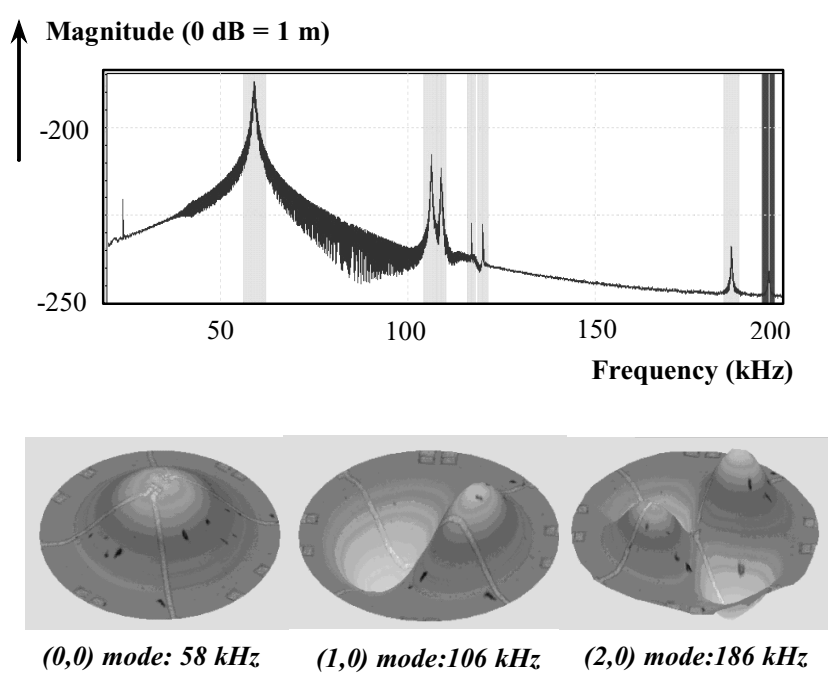

Figure 6: Average spectrum and visualization of the vibrating diaphragm mode shapes using a scanning laser vibrometer.

Figure 6 shows the average spectrum of the diaphragm surface response to the excitation signal and also illustrates the first three vibration modes.

\section{Acoustic Characterization - Receiver}

The dynamic response of the sensor to acoustic pressure perturbations was characterized in a plane wave tube (PWT). The PWT consists of a rigid-walled duct that supports planar $(0,0$ mode) acoustic waves propagating along the length of the duct. Thus, sensors placed at the same axial location from the acoustic driver sense the same acoustic pressure field.

The sensors were calibrated in two different PWTs, a $25.4 \mathrm{~mm}$ x $25.4 \mathrm{~mm}$ normal incidence PWT and an $8.5 \mathrm{~mm} \times 8.5 \mathrm{~mm}$ grazing incidence PWT. The sensor and a reference microphone (Brüel and Kjær Type 4138) were flush mounted at the same axial distance from the acoustic driver. The sensor was biased at $9 \mathrm{~V}$, and the differential output of the Wheatstone bridge was connected to a SR560 preamplifier. The amplified signal was then fed into a SR785 dynamic spectrum analyzer for data processing.

The low-frequency cut-off for the first non-planar $(1,0),(0,1)$ mode is $20 \mathrm{kHz}$ for the grazing incidence PWT and $6.7 \mathrm{kHz}$ for the normal incidence tube. Thus, the useable bandwidth is limited to $20 \mathrm{kHz}$ and $6.7 \mathrm{kHz}$ respectively. A low frequency limit of $1 \mathrm{kHz}$ is imposed by the compression driver, which becomes excursion limited at low frequencies.

\section{Linearity}

The normal incidence PWT was used to measure the linear response of the sensor to varying sound pressure levels. A $1 \mathrm{kHz}$ tone at varying amplitudes, monitored by the reference microphone, was used to excite the sensor, which was biased at $9 \mathrm{~V}$. The rms output voltage of the sensor at each sound pressure level was recorded. The output voltages are shown in Figure 7 for sound pressure levels up to $140 d B$ (ref $20 \mu P a$ ). The results indicate a linear response to acoustic pressure perturbations over four orders of magnitude $(60-140 d B)$. The calculated sensitivity at $1 \mathrm{kHz}$ is $1.415 \pm 0.0002 \mu \mathrm{V} /(\mathrm{V} . \mathrm{Pa})$, or equivalently, $-117 \mathrm{~dB}$ re $1 V /(V . P a)$.

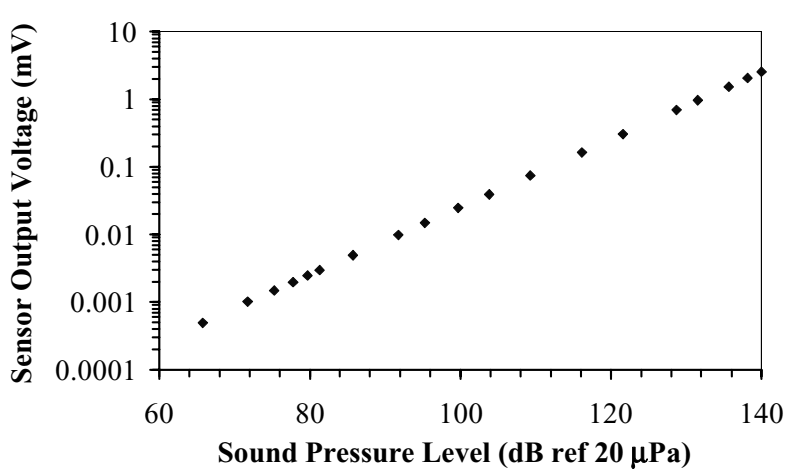

Figure 7: Plot illustrating the device linearity in sensing acoustic pressure perturbations (up to $140 \mathrm{~dB}$ ) at a frequency of $1 \mathrm{kHz}$.

\section{Frequency Response}

Figure 8 shows the magnitude frequency response of the sensor calibrated in the grazing incidence PWT, as well as the normal incidence tube using a constant amplitude tone of $110 \mathrm{~dB}$. The plot indicates a flat frequency response with an average sensitivity of $0.98 \mu V /(V . P a)$ or equivalently $-120.2 d B$ re 1 $V /(V . P a)$ with a standard deviation of $1 d B$ over measured frequency range.

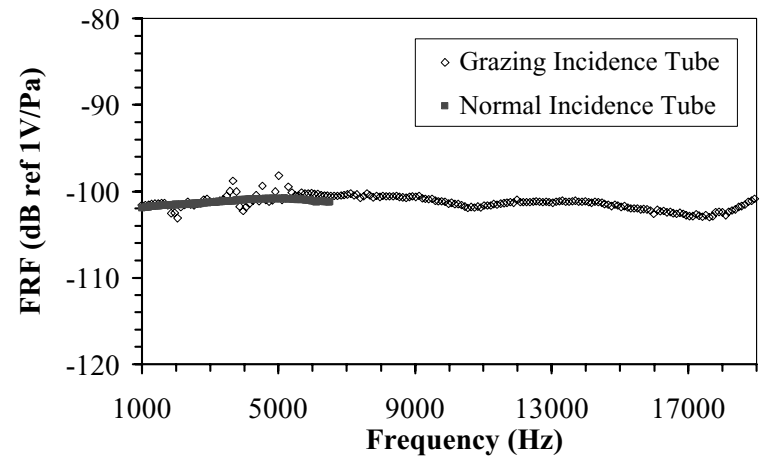

Figure 8: Magnitude of the sensor frequency response function to a constant sound pressure level of $110 \mathrm{~dB}$.

The results obtained from the grazing incidence PWT indicate data scatter at low frequencies. These variations may be attributed to the non-ideal propagation characteristics of the tube, including a compliant boundary condition and an area change at the tube termination. The same frequency range $(1-6 \mathrm{kHz})$ tested in the normal incidence tube indicates a flat sensor response.

\section{Acoustic Characterization - Transmitter}

The end application requires an array of sensors to be used in a network to monitor the state of the entire cavity. When used as an array, minimal cross-talk between the sensors is required for efficient real-time monitoring. The directional behavior of the generated acoustic field and the spacing between the sensors will determine the cross-talk. The acoustic field generated by the vibration of the thermoelastically actuated diaphragm was characterized in a free-field environment. In order to characterize the transmitted acoustic field, two sets of measurements were obtained. In both cases, the transmitting sensor was fixed and oriented such that the diaphragm surface is vertical. The transmitter was excited with a combination of harmonic ac voltage $(9 \mathrm{Vpk})$ and a dc $(9 \mathrm{~V})$ voltage. The acoustic field was measured using a Brüel and Kjær Type 4138 condenser microphone. To measure the directionality of the generated acoustic field, the microphone was positioned at a fixed radial distance of $25 \mathrm{~mm}$ 
from the transmitter and a jig was constructed that allowed the microphone to be revolved around the transmitter at the fixed radius. Sound pressure measurements were made at $2^{\circ}$ intervals, from $0^{\circ}$ (on axis) to $90^{\circ}$, averaged, and recorded.

To obtain the variation of sound pressure level with distance from the transmitter, the microphone was positioned directly opposite the transmitter on a single axis traverse with a precision of 1 micron. The initial position was set at $3 \mathrm{~mm}$ from the transmitting diaphragm and then varied to $50 \mathrm{~mm}$ along the axis of the diaphragm. At each position, sound pressure measurements were obtained using a SR785 dynamic spectrum analyzer.

Figure 9 shows the normalized directional response of the generated acoustic field, which indicates a drop in sound pressure level with increasing angle from the diaphragm axis. At an angle of $90^{\circ}$ the sound pressure level is reduced to $40 \%$ of the maximum on-axis value. Theoretically, a sensor with Helmholtz number $k a<1$ should exhibit omni-directional radiation pattern, where $k$ is the wave number given by $\omega / c$ and $a$ is the diameter of the diaphragm. The observed difference between theory and experiment may be attributed to the packaging of the sensor, which is recessed in a square depression of $0.5 \mathrm{~mm}$, or the statically deflected diaphragm, which may also have some effect on focusing the sound field. The oscillations in sound pressure level with changing angle may be the result of scattering from the sensor package.

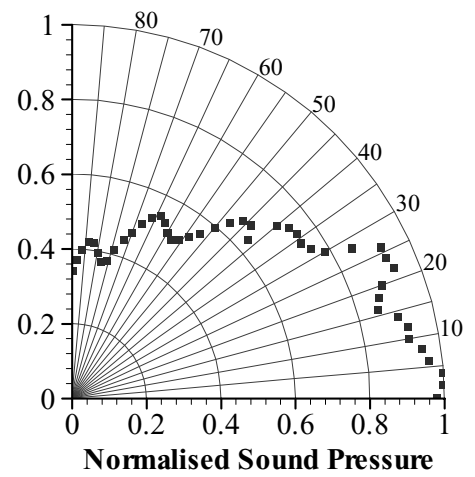

Figure 9: Directivity of the generated acoustic field at a frequency of $60 \mathrm{kHz}$.

Sound pressure measurements versus distance from the diaphragm surface are plotted in Figure 10.

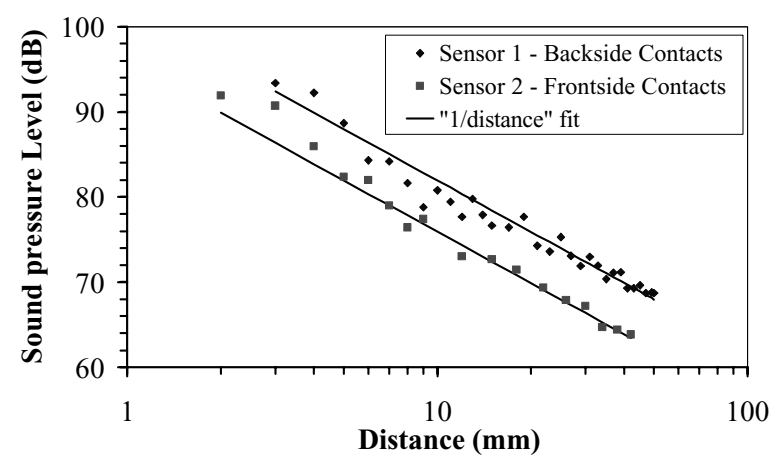

Figure 10: Sound pressure level of generated acoustic field as a function of radial distance, at a frequency of $60 \mathrm{kHz}$.

The graph indicates a transmitting sensitivity of $22 \mathrm{mPa} / \mathrm{V}$ at a distance of $10 \mathrm{~mm}$ and an operating frequency of $60 \mathrm{kHz}$. The plot also shows the inverse relation between sound pressure level and distance. The oscillations in the data are due to scattering from the microphone surface resulting in the formation of a standing wave between the transmitter and the microphone. The scattering effect is reduced with increasing distance from the transmitter.

The effect of varying static and dynamic power used to excite the sensor at a fixed frequency $(55 \mathrm{kHz})$ was also investigated.

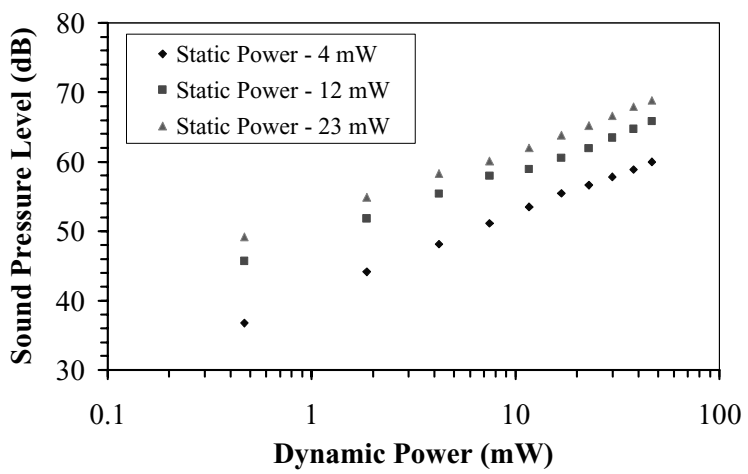

Figure 11: Variation in sound pressure level with change in static and dynamic input power.

The results are plotted in Figure 11 and indicate a direct dependence on the dynamic power. The sound pressure level also increases with increasing static power for a given dynamic power. The low sound pressure level observed even at high excitation voltage is due to the operating frequency. The device has a high quality factor (see Figure 6) and exhibits appreciable response only near its resonant frequency.

\section{Noise Floor Spectra}

The study of the electrical noise floor of a device is required to determine the minimum detectable signal (MDS). Measurements of the noise power spectral density (PSD) were made in a Faraday cage using low-noise test equipment. The aim of the experimental setup is to isolate the random physical noise of the device under test (DUT) from deterministic interference. Deterministic sources arise from capacitive coupling of electromagnetic interference (EMI) to the device and cabling, with the ac power line being the major contributor (60 $\mathrm{Hz}$ and its harmonics). The Faraday cage considerably reduces the interference, permitting analysis of the noise PSD.

The sensor was configured similar to the operating conditions with a $9 \mathrm{~V}$ bias across the Wheatstone bridge. A battery powered Stanford Research Systems SR560 differential amplifier with specified noise voltage of $4 \mathrm{nV} / \sqrt{ } \mathrm{Hz}$ was used to amplify the differential voltage from the bridge with a gain of 10,000. After proper grounding of all measurement equipment, the noise PSD was measured using a SR785 dynamic spectrum analyzer using 500 averages. In order to maintain sufficient resolution at low frequencies, a low frequency range of $0-1.6 \mathrm{kHz}$ with a $2 \mathrm{~Hz}$ bin was used. This frequency resolution effectively isolates the $60 \mathrm{~Hz}$ power line interference. Larger frequency ranges of $25.6 \mathrm{kHz}$ with $8 \mathrm{~Hz}$ bin were used for the higher frequencies. In order to extract the device noise floor, the set-up noise PSD was measured by shorting the differential outputs of the bridge and was subtracted from the total noise PSD. The MDS (per $\sqrt{ } \mathrm{Hz}$ ) was then calculated by taking the square root of the noise PSD and dividing with the sensitivity of the device to obtain pressure. 


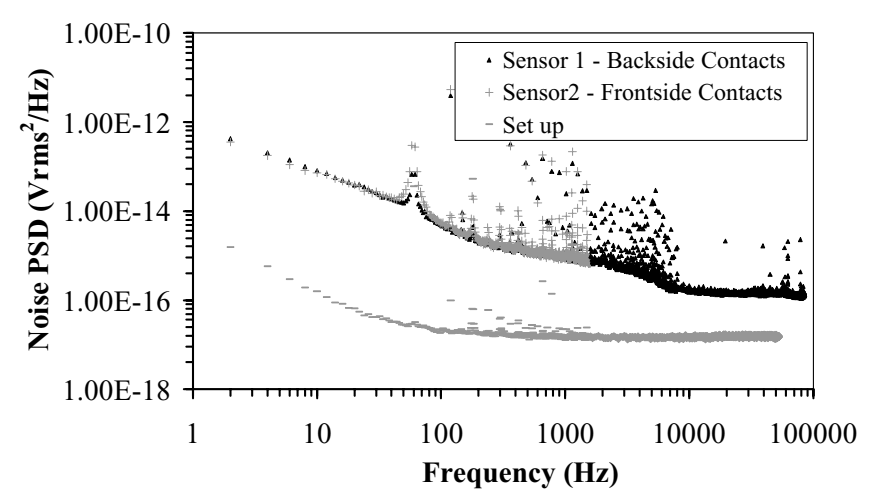

Figure 12: Noise power spectral density of the sensor at a bridge bias of $9 \mathrm{~V}$ (set-up noise included).

The voltage noise PSD of the sensor is plotted in Figure 12. As indicated, the voltage noise PSD is dominated by $1 / f$ noise at low frequencies. Additional plots of the set-up noise from the amplifier and EMI and a sensor with front-side contacts are also shown for comparison. The results indicate a negligible noise contribution from the interconnect. The $1 / f$ noise intersects the thermal noise $\left(\approx 1.4 \times 10^{-16} \mathrm{~V}^{2} / \mathrm{Hz}\right)$ at approximately $10 \mathrm{kHz}$, making the device only Johnson noise limited at the operating frequencies. The "spikes" in the data are due to the deterministic interference at $60 \mathrm{~Hz}$ and $20 \mathrm{kHz}$ and their harmonics.

\section{PROXIMITY SENSING}

To demonstrate the use of the device as a proximity sensor, two sensors were placed $20 \mathrm{~mm}$ apart and oriented towards each other. The transmitting sensor was electrothermally excited using a $9 \mathrm{Vpk}$ ac signal over a $10 \mathrm{kHz}$ frequency range. The generated acoustic signals were detected using a second sensor as the receiver. The receiver was selected such that its first resonance is higher than that of the transmitter. Figure 13 shows the normalized frequency response of the receiver compared to the response of the reference microphone (Brüel and Kjær Type 4138). The plot indicates good agreement between the sensor and the reference microphone. The deviation in response beyond $62 \mathrm{kHz}$ is due to the fact that the receiving sensor is approaching its own resonance near $70 \mathrm{kHz}$.

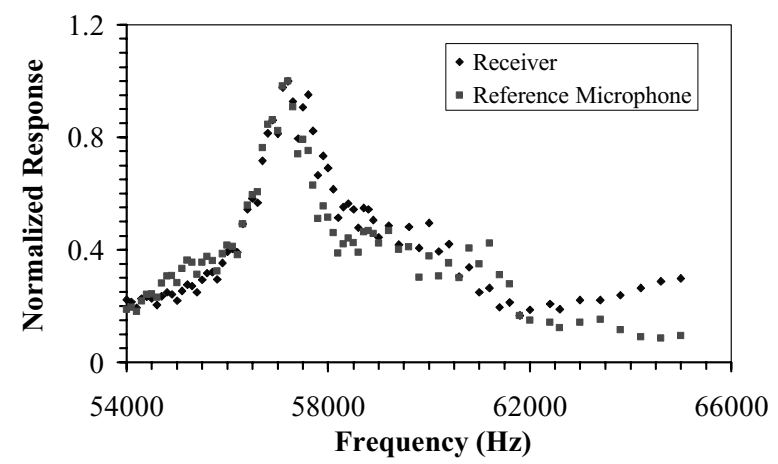

Figure 13: Plot illustrating the frequency response of the receiver compared to a reference microphone.

\section{CONCLUSIONS AND FUTURE WORK}

An acoustic proximity sensor with integrated through-wafer electrical interconnects has been developed. Preliminary characterizations indicate a flat frequency response, good acoustic receiving sensitivity and a low noise floor (1.34 $\mathrm{mPa} / \sqrt{ } \mathrm{Hz}-\mathrm{MDS})$.

Future work will focus on a more thorough characterization of the sensor and the development of an optimized $2^{\text {nd }}$ generation transmitter/receiver based on a non-linear, composite, dynamic model of the diaphragm. The sensor will be implemented with different proximity sensing algorithms developed for real-time monitoring of the cavity surrounding the HSSV. Also, detailed characterization of the diaphragm surface response to various excitation signals using the scanning laser vibrometer will be performed. This information will be used to project the acoustic field generated by the diaphragm via Rayleigh's integral and compared to the acoustic measurement of the radiation pattern.

\section{ACKNOWLEDGEMENT}

This work is supported by the Office of Naval Research (contract \#N00014-00-1-0343) monitored by Dr. Kam Ng. The sensors were fabricated in the Stanford nanofabrication facility at the Center for Integrated Systems, Stanford.

\section{REFERENCES}

1. D. J. Warner, "Technology Assessment of Hydrodynamic/ Supercavitating Technologies," Alliant Techsystems Inc. Final Report, June 1998.

2. M. Rossi, "Acoustics and Electroacoustics," Artech House, 1988.

3. M. R. Hornung, and O. Brand, "Micromachined UltrasoundBased Proximity Sensors," Kluwer Academic Publishers, Boston, 1999.

4. X. Jin, I. Ladabaum, F. L. Degertekin, et. al., "Fabrication and Characterization of Surface Micromachined Capacitive Ultrasonic Immersion Transducers" JMEMS, vol. 8, No. 1, pp. 100-14, 1999.

5. J. Bernstein, K. Houston, L. Niles, et. al., "Micromachined Ferroelectric Transducers for Acoustic Imaging, "Transducers '97, pp. 421-4, 1997.

6. E. M. Chow, A. Partridge, C. F. Quate, et. al., "ThroughWafer Electrical Interconnects Compatible with Standard Semiconductor Processing," Solid State Sensors and Actuator Workshop, pp.343-6, 2000.

7. V. Chandrasekaran, E. M. Chow, T. W. Kenny, et. al., "Through Wafer Electrical Interconnects For MEMS Sensors," ASME 2001, New York, 2001.

8. E.M. Chow, H.T. Soh, and H.C. Lee, "Integration of throughwafer interconnects with a two-dimensional cantilever array," Sensors and Actuators A, vol. A83, pp.118, 2000.

9. J. N. Reddy, "Mechanics of Laminated Composite Plates: Theory and Analysis," CRC Press, Boca Raton, FL, 1997.

10. M. Ozisik, "Heat Conduction," Wiley-Interscience Publication, pp. 2, 2000.

11. M. Sheplak, K. S. Breuer, and M. A. Schmidt, "DielectricallyIsolated, Single-Crystal Silicon, Piezoresistive Microphone," Solid-State Sensor and Actuator Workshop, pp. 23-6, 1998.

12. M. E. Law and S. Cea, "Continuum Based Modeling of Silicon Integrated Circuit Processing: An Object Oriented Approach," Computational Materials Science, vol. 12, No. 1, pp. 289-308, 1998. 